\title{
De l'apprentissage du commentaire de la peinture à celui de la littérature : la possibilité d'un détour pédagogique?
}

\author{
Marie-Sylvie Claude \\ ESCOL-CIRCEFT, ESPE de Créteil, UPEC, Créteil, France
}

\begin{abstract}
Résumé. Cette contribution s'inscrit dans le champ plus vaste d'un travail de thèse, dont nous exposons d'abord rapidement la problématique afin d'y situer l'objet principal de l'article, la comparaison de deux commentaires écrits par la même élève, l'un sur un tableau de peinture et l'autre sur un texte littéraire.
\end{abstract}

\section{Situation dans la recherche globale}

\subsection{Problématique}

L'expression commentaire de la peinture n'apparaît pas dans les programmes de français du collège et du lycée (nous nous intéressons aux classes de troisième et de seconde, 15-16 ans). C'est l'expression lecture de l'image qui désigne, dans les programmes du secondaire, dans les rapports d'inspection et dans les manuels scolaires, l'activité scolaire sur laquelle nous travaillons. Si le mot image pose problème (toute image ne produit pas le sens de la même façon; toute peinture ne fait pas image), c'est sur le mot lecture surtout que nous nous pencherons.

La lecture de l'image est ainsi décrite dans les programmes : «Dans une démarche comparable à la lecture des textes, l'image est analysée en tant que langage (...). Face à l'image, comme face au texte, les élèves doivent apprendre à s'interroger sur ce qu'ils voient et à observer l'image avant d'en parler. On pourra alors les amener à passer d'une approche intuitive à une interprétation raisonnée en les initiant progressivement à quelques notions d'analyse. ${ }^{1}$ L'exercice se définit donc par la comparaison avec les exercices de lecture littéraire, plus précisément avec la lecture analytique, ainsi définie : « La lecture analytique se définit comme une lecture attentive et réfléchie, cherchant à éclairer le sens des textes et à construire chez l'élève des compétences d'analyse et d'interprétation. Elle permet de s'appuyer sur une approche intuitive, sur les réactions spontanées de la classe, pour aller vers une interprétation raisonnée. $»^{2}$ Les mêmes termes sont utilisés pour la littérature et pour l'image : on peut donc en déduire que ce qui est attendu des élèves est du même ordre, qu'ils doivent apprendre à passer progressivement d'une « approche intuitive », spontanée, à une « interprétation raisonnée » appuyée sur une analyse de l'objet. Que conclure de ce parallèle ? Les programmes partent du principe qu'il est possible d'attendre des élèves la même prestation sur les deux arts (si l'on entend par lecture le discours interprétatif sur

\footnotetext{
${ }^{1}$ Programmes du collège. Programmes de l'enseignement du français. Bulletin officiel spécial n` 6 du 28 août 2008 . Page 3.

$2 \mathrm{Ib}$ pages 2 et 3 .
}

This is an Open Access article distributed under the terms of the Creative Commons Attribution License 4.0, which permits unrestricted use, distribution, and reproduction in any medium, provided the original work is properly cited. 
l'objet). Mais ils présupposent aussi que les opérations intellectuelles pour arriver à cette prestation sont les mêmes (le mot lecture désignant aussi l'opération intellectuelle qui permet de décoder et de construire le sens). L'emploi du même mot lecture implicite donc entre les deux activités une double isomorphie, ce qui présente comme évidemment possible la construction de compétences considérées comme communes aux deux arts par le biais d'un travail sur l'un ou l'autre de ces deux arts.

Or au sens propre on ne lit que les textes. La peinture, qui ne signifie pas par le truchement d'une langue, fait sens de façon très différente de la littérature. Le travail du récepteur n'est pas le même puisque s'il infère du sens des caractéristiques formelles dans les deux cas, il en infère de la peinture sans l'appui d'un sens littéral. Les connaissances culturelles nécessaires sont différentes car les codes iconographiques ne sont pas les codes linguistiques, même si certaines connaissances (sur le contexte, sur le mouvement...) sont communes. On peut donc se demander si la double isomorphie présupposée par les programmes et le détour pédagogique auquel elle invite ne relève pas d'une doxa.

C'est pourquoi nous nous interrogeons sur la part de fondement de ce postulat de la scolarisation en parallèle des deux arts et sur les conditions et les limites du détour pédagogique. Je m'inscris pour ce faire dans le cadre théorique des travaux de l'équipe ESCOL sur la constitution des objets du monde en objets d'étude, en contexte scolaire, et sur les difficultés que cette opération est susceptible de générer pour une partie des élèves.

\subsection{Situation dans cet ensemble de la comparaison des commentaires d'élèves}

Pour mieux connaître les attendus des enseignants et leurs pratiques en lecture de la peinture, nous avons interrogé des professeurs de français de l'académie de Créteil (150 questionnaires et une vingtaine d'entretiens). Nous avons cherché à connaître leurs pratiques, leurs difficultés, et leur perception du lien entre lecture de la littérature et lecture de la peinture. Ceux que nous avons interrogés semblent considérer l'isomorphie comme allant de soi et pratiquer avec leurs élèves le même type d'activité de lecture analytique ou decommentaire sur la peinture que sur la littérature, en vue de travailler des compétences communes d'interprétation. Ainsi déclarent-ils par exemple qu'ils cherchent à initier leurs élèves, grâce à un passage par la peinture, au processus qui consiste à repérer une caractéristique formelle, à l'analyser pour en déduire des effets de sens et à mettre en mots ces effets de sens. Les élèves ayant compris comment construire le sens de tel choix plastique dans un tableau de peinture pourraient transposer cette compétence à la littérature et ainsi donner du sens à tel choix stylistique. Dans leur grande majorité les enseignants que j'ai interrogés considèrent que leurs élèves sont plus à l'aise dans les exercices d'interprétation de la peinture que dans ceux de lecture littéraire, qui les mettent fréquemment en difficulté, et sont donc nombreux à plébisciter ce passage par la peinture. Nous avons cherché à comprendre précisément, au travers de leurs réponses, quels étaient les apprentissages qu'ils visaient et donc quelles étaient leurs attentes, in fine, pour cet exercice de lecture interprétative, sur l'un et l'autre des deux arts : pour résumer les réponses que nous avons obtenues, ils souhaitent que leurs élèves soient capables d'aller au-delà de la compréhension immédiate afin de construire un sens pluriel, sens inféré des caractéristiques formelles et informé par la prise en compte de données historiques et esthétiques ; ce sens pluriel ne se superpose pas nécessairement avec les intentions de l'artiste.

La satisfaction de ces attendus nécessite une activité complexe. Le modèle théorique des registres d'intellection tel qu'il est actuellement travaillé par l'équipe $\mathrm{ESCOL}^{3}$, modèle qui définit le travail scolaire comme constitué de l'intersection de trois registres d'intellection, cognitif, culturel et symbolique, nous en a fourni un outil d'analyse pertinent : l'activité d'intellection permettant l'accès à un registre scolaire littératié se définit par un équilibrage complexe d'opérations cognitives et de connaissances culturelles, sous tendues par une représentation spécifique de l'œuvre d'art (littéraire ou

\footnotetext{
${ }^{3}$ Bautier, E., Rayou, P. Les difficultés d'apprentissages Les inégalités d'apprentissage. Programmes, pratiques et malentendus scolaires. (Nouvelle édition 2013) Paris : PUF.
} 
picturale $)^{4}$, représentation naturalisée par les enseignants mais qui relève d'une construction et qui n'est pas partagée au-delà d'une communauté lettrée. Ce registre scolaire littératié suppose la mobilisation de savoirs scientifiquement adéquats aux disciplines de référence, par des références culturelles légitimes, et, pour ce que nous appelons le registre symbolique, par l'appartenance au « discours vertical »'une communauté savante institutionnelle.

Pour savoir si l'accès au registre scolaire adéquat est effectivement favorisé par un travail sur la peinture, nous avons constitué un corpus de commentaires écrits par les mêmes élèves sur un tableau de peinture d'une part et sur un texte littéraire d'autre part. Nous avons obtenu de 106 élèves de trois classes de troisième et de trois classes de seconde de l'académie de Créteil un court commentaire sur un tableau et un court commentaire sur un texte littéraire. Chaque élève a travaillé sur un des trois extraits littéraires suivant : un extrait de Sido Colette (1929), un extrait d'Orphée de Jean Cocteau (1927); «Le Pont Mirabeau »de Guillaume Apollinaire, dans Alcools (1913) ; et sur une des trois reproductions suivantes : La Crucifixion blanche de Marc Chagall (1938); La Famille de Saltimbanques, de Picasso (1905) ; Paysage sous la pluie, de Vassily Kandinsky (1913). Les élèves disposaient chacun d'une lettre expliquant le contexte de la recherche, d'une reproduction couleur et d'une photocopie d'un des extraits ; la consigne était la même sur les deux objets : Ecrivez dix lignes (au minimum) pour commenter le texte [le tableau] (donnez quelques unes de vos impressions). Ils ont travaillé en classe sous la surveillance de leur enseignant de français pendant une heure trente.

L'analyse détaillée de certains commentaires d'élèves nous a amenée à faire apparaître progressivement des critères définissant le mode d'investissement plus ou moins littératié de chacun des registres et de définir des caractéristiques langagières permettant de classer les commentaires d'élèves selon chacun de ces critères.

\section{Analyse comparative des commentaires de Laura}

Nous proposons ici un exemple de cette analyse comparative, celle des commentaires de Laura, élève de troisième au collège Schweitzer de Créteil, qui a travaillé sur le texte de Colette et sur le tableau de Kandinsky :

\section{Colette.}

Le texte est assez difficile à comprendre, il faut le lire et le relire pour comprendre un peu mieux de quoi il s'agit. Dans ce texte Colette donne son avis sur le monde dans lequel elle vit. Tout le texte est fait dans la description, il y a beaucoup d'adjectifs qualificatif. Colette donne son avis sur tous ce qu'elle a vécu et ce qu'elle a vu comme lorsqu'elle dit "Elle se décourageait aussitôt née et replongeait dans la terre ». Elle se met à la place des choses ou des personnes autour d'elle.

Le texte pourrait parler de la période de l'enfance ou tout paraît magique mais d'un côté le texte évoque les choses d'une manière que seul les adultes peuvent évoquer.

Le texte est très difficile à cerner et à décrire surtout qu'il y a énormèment de mots soutenu.

Vassily Kandinsky. Paysage sous la pluie. 1913.

Ce tableau est multicolore et la façon dont sont mise les couleurs me font penser à un monde horrible et étoufant. Dans ce tableau la couleur noire en haut à gauche me fait penser à un animal ou à quelque chose d'éfrayant. Il y a aussi comme des grilles qui me font penser à l'enfermement comme si le peintre pensait que la vie ressemblais à la prison et que l'on est pas libre. Le bleu me fait penser à de l'eau et le rouge au sang ou aussi au ciel lorsque nous somme près d'un volcan en éruption.

Je vois dans ce tableau une ville avec plein de grille sous un ciel rouge avec la mer qui recouvre la ville et quelqu'un ou quelque chose qui approche de cette ville horrible.

${ }^{4}$ Bernstein, B. Pédagogie, contrôle symbolique et identité. Théorie, recherche, critique. (2007/1996 pages 227-253), Laval, PUL. 
Le peintre devait être dans un état d'esprit triste ou encore ennervé car quand on est souvent ennervé on voit le monde comme s'il était cruel. Mais cette idée me fait voir dans le tableau la mort par le rouge du tableau. On dirait aussi que tout détein sur les différents éléments. ${ }^{5}$

\subsection{Comparaison du registre cognitif}

Les résultats de l'enquête auprès des enseignants d'une part et nos lectures du côté disciplines de référence (sémiologie, histoire de l'art, didactique de la lecture littéraire) d'autre part nous ont permis de définir les critères selon lesquels on considérera ce registre de niveau plus ou moins littératié : la posture interprétative, plus ou moins adéquate selon que le commentaire de l'élève correspond à une pure restitution du sens immédiat ou à la conception par le sujet d'un surplus de sens ; la prise en compte plus ou moins opérationnelle des caractéristiques de la forme comme lieux de production du sens ; l'appréhension plus ou moins pertinente de l'objet comme un système signifiant complexe et cohérent ; enfin, la perception plus ou moins riche du sens comme pluriel. Ces différents critères sont évidemment étroitement liés : si nous en déconstruirons ici l'enchâssement dans les commentaires de l'élève, c'est pour la clarté de la démarche comparative.

\subsubsection{La qualité de la posture interprétative}

Le commentaire de Laura démarre sur l'expression claire d'un ressenti : monde horrible et étoufant, que la suite s'efforce d'étayer. La présence croisée des marques du sujet de l'énonciation et des références au «peintre », au «tableau », montre que l'auteur du commentaire fait du tableau son objet de travail (comme si le peintre pensait ; le peintre devait être ; je vois dans ce tableau...). Elle ne se contente pas de décrire ce qui est visible : la formule réitérée me fait penser à signale la construction d'un surplus de sens. Le passage d'un vocabulaire concret à un vocabulaire abstrait (état d'esprit triste, ennervé) marque également un déplacement de l'immédiatement visible à des effets de sens symboliques, déplacement caractéristique de la démarche interprétative.

La phrase liminaire du commentaire sur le texte de Colette contraste avec celle qui concerne le tableau puisqu'il s'agit d'un aveu d'incompétence (difficile à comprendre). La dernière phrase y reviendra (Le texte est très difficile à cerner et à décrire). Entre ces deux expressions de la difficulté, l'élève semble s'engager dans l'interprétation en encadrant une citation du texte de remarques personnelles s'en abstrayant (l'auteur donne son avis sur le monde (...) sur tous ce qu'elle a vécu et qu'elle $a v u$ etc.). Mais quand on y regarde de près il apparaît que cette alternance, apparemment bien dominée sur le plan langagier n'est que la marque factice - relevant sans doute d'un procédural scolaire appris - d'une orchestration de la polyphonie certes caractéristique de cet exercice, mais qui n'est pas effective. En effet, la citation qui est à l'appui de ces remarques n'a à peu près rien à voir avec elles (à tout prendre n'importe quelle phrase du texte - de n'importe quel texte même ! - aurait pu faire l'affaire). De sorte que les remarques qui pourraient relever de l'interprétation apparaissent comme des formules toute faites plaquées sur le texte : on pourrait dire de tout auteur qu'il donne son avis sur le monde.

Une autre différence essentielle de ce commentaire d'élève avec celui qu'elle a produit sur la peinture est l'absence complète de toute marque explicite de la subjectivité : aucune occurrence de la première personne, qui saturait le texte sur Kandinsky, aucune mention de la formule me fait penser à (contre quatre occurrences sur Kandinsky, sans compter des quasi-équivalences : cette idée me fait voir, on dirait que). N'étant pas nourrie de réaction subjective, l'interprétation semble tourner à vide.

\footnotetext{
${ }^{5}$ L'orthographe originale de l'élève a été reproduite.
} 


\subsubsection{La prise en compte des caractéristiques de la forme}

Sur le tableau de Kandinsky le commentaire va et vient entre la mention des caractéristiques plastiques, couleurs et traits, et l'interprétation : repérage et interprétation symbolique de la multiplicité (multicolore) des couleurs prises globalement dans la façon dont [elles]sont mise, puis ciblage sur la couleur noire liée à quelque chose d'effrayant, le bleu lié à l'eau puis à la figure de l'inondation, et enfin le rouge, couleur sur laquelle revient la fin du commentaire, interprétée comme symbole du sang, du ciel menaçant (un volcan en éruption), puis de la mort. Par ailleurs, le graphisme rectiligne est identifié comme une grille, et interprété comme un symbole de l'enfermement.

Le passage de la forme au sens est cependant, à plusieurs reprises, peu étayé : par exemple le thème de la mort est seulement justifié par la couleur rouge, ce qui paraît peu convaincant (cette idée me fait voir dans le tableau la mort par le rouge du tableau). C'est le cas aussi de certaines identifications iconiques des signes plastiques, qui pour ce tableau sont déjà des interprétations. Rien ne justifie par exemple que la couleur noire en haut à gauche puisse être interprétée comme l'image d'un animal, ni que quelqu'un ou quelque chose menace la ville, dont l'identification comme telle n'est pas non plus justifiée. Pour qu'il y ait véritablement ressaisie du ressenti, donc accès à un niveau littératié, une meilleure justification de l'interprétation serait nécessaire de façon à ce que le lecteur puisse la considérer comme recevable.

Sur le texte de Colette, la forme semble prise en compte avec la mention de la description et des adjectifs qualificatifs. Mais si la phrase Colette donne son avis sur tous ce qu'elle a vécu et ce qu'elle a $v u$ pourrait ressembler à une inférence interprétative issue de l'analyse de la description et des adjectifs, la citation proposée en guise d'illustration ne présente ni adjectifs qualificatifs ni éléments descriptifs ( Elle se décourageait aussitôt née et replongeait dans la terre »). Cette citation, qui serait surtout intéressante par la personnification qu'elle contient, est suivie d'une phrase (elle se met à la place des choses ou des personnes autour d'elle) qui pourrait constituer une interprétation mais qui fait problème : justement la narratrice est seule, sa solitude matinale est même un leit motiv du texte ; et la personnification signifierait plutôt qu'elle met les éléments inanimés à la place des animés. L'avant dernière phrase du commentaire ouvre une piste plus adéquate aux attendus : Le texte pourrait parler de la période de l'enfance où tout paraît magique mais d'un côté le texte évoque les choses d'une manière que seul les adultes peuvent comprendre. Le contraste perçu entre le contenu (la magie de l'enfance) et la forme de son expression (résolument adulte) pourrait relever d'un registre cognitif de bon niveau mais l'entreprise tourne court car l'élève revient à l'expression de son incompétence.

\subsubsection{L'appréhension de l'objet comme un tout complexe et cohérent}

Sur le texte de Kandinsky, les signes plastiques et certains assemblages plastiques perçus comme des signes iconiques sont croisés selon ce qu'ils symbolisent pour l'auteur du commentaire. Des réseaux signifiants se construisent ainsi : celui de l'enfermement, annoncé dès la phrase liminaire au travers du mot étoufant puis inféré de la couleur noire qui fait naître l'image d'un animal en cage, repris par le motif de la prison, motif élargi à une allégorie de la vie (comme si le peintre pensait que la vie ressemblais à la prison). Un autre réseau articulé sur le premier s'établit autour des figures effrayantes : «quelque chose d'éfrayant», «quelqu'un ou quelque chose qui approche ». Le paradigme des figures apocalyptiques s'y greffe, eau et feu destructeurs : volcan en éruption, mer qui recouvre la ville, ville horrible. Les figures du sang et de la mort s'articulent logiquement sur l'ensemble. La cohérence du texte est satisfaisante puisque l'articulation enfermement/angoisse/apocalypse/mort fédère l'ensemble de l'interprétation, annoncé en introduction par les mots horrible et étoufant et repris en fin de texte par le schème d'un monde cruel et morbide.

Dans la dernière phrase du commentaire, une intéressante observation plastique est proposée (tout détein sur les différents éléments), qui n'est pas intégrée dans le tissage interprétatif : on pourrait assez facilement guider l'élève pour rester dans la logique de sa réception vers l'interprétation de ce débordement des formes par les couleurs comme le signe de l'envahissement de l'angoisse. 
Sur Colette, on ne perçoit pas d'autre fil rouge que l'aveu d'incompétence. Nous avons vu que l'alternance entre citation ou références au texte et remarques prises en charge par le sujet de l'énonciation relevait de la juxtaposition et non de l'articulation logique. La parataxe et l'absence de connecteurs marquent cette cohérence défaillante, alors que le commentaire sur Kandinsky utilise largement les liens syntaxiques.

\subsubsection{La perception du sens comme pluriel}

Sur le texte de Kandinsky, la recherche d'une interprétation plurielle est marquée par des formules alternatives : ou à quelque chose d'éfrayant, ou aussi au ciel.... Certaines formules manifestent en outre la conscience de la subjectivité de l'interprétation, qui se présente ainsi comme un possible ouvert à d'autres possibles (comme si le peintre pensait, le peintre devait être, on dirait que ; il y a aussi comme des grilles). On perçoit même l'émergence d'une structuration possible du discours interprétatif selon deux grandes directions de sens : interprétation du tableau comme représentation apocalyptique d'abord ; puis comme projection de l'angoisse du peintre. Ceci pourrait être le plan d'un commentaire dont ce texte serait un (tout) premier jet.

Sur Colette rien ne permet de conclure à la perception du sens comme potentiellement polysémique.

On peut conclure que sur Kandinsky la posture interprétative de niveau littératié est en cours d'acquisition, même si elle n'est pas vraiment (ou pas encore ?) opérante, la réception proposée restant peu universalisable faute d'une justification suffisante qui, même si elle est forcément contestable, permettrait de l'objectiver (au sens où elle s'abstrairait de la pure décision d'une subjectivité pour devenir objet communicable). L'élève pourrait être guidée dans une réécriture de ce premier jet par le biais d'un étayage s'attachant à lui faire percevoir cet écueil.

Sur Colette en revanche, des procédures scolaires semblent mobilisées sans qu'elles soient nourries par le contenu interprétatif qu'elles devraient avoir pour fonction de mettre en forme. L'absence d'implication subjective peut être une explication de ce niveau peu littératié du registre cognitif.

Mais pourquoi cet investissement hétérogène des deux objets ? Il nous semble que pour étayer une réécriture de ces commentaires qui le rende plus conforme aux attentes scolaires, pour accéder à un registre plus littératié, le seul réajustement du registre cognitif ne suffirait sans doute pas : le défaut de communicabilité de l'interprétation du tableau de Kandinsky et le défaut de ressenti à ressaisir sur le texte de Colette sont des défaillances qui ne concernent pas seulement le cognitif mais tiennent aussi des autres registres d'intellection, qui lui sont étroitement liés et dont nous poursuivons la déconstruction pour comparer l'activité déployée par l'élève sur chacun des deux objets.

\subsection{Comparaison du registre culturel}

Sur Kandinsky, les topoï de l'éruption volcanique, du ciel rouge et du déluge sont à l'appui de l'interprétation : angoisse de l'enfermement et menace mystérieuse. Ces références, présentes dans les mythologies païennes ou bibliques, relèvent d'une culture légitime, ce qui pourrait contribuer à rendre possible le partage avec tout autre de cette interprétation. Mais ceci nous semble filtré par un univers de références juvéniles qui lui-même exploite des références à la culture légitime (le déluge, l'animal monstrueux, la menace invisible et mystérieuse... sont aussi des éléments d'un fantastique convenu largement exploité notamment dans les jeux vidéo ou dans les mangas contemporains). La présence de la mort et de l' angoisse semble en effet apparaître à cette élève comme évidente dans ce cadre culturel, de sorte qu'elle la justifie peu, et que la valeur symbolique des éléments cités, qui sont in fine peu exploités dans le commentaire, en est appauvrie. On peut penser que pour revivifier ces références, connues dans un seul de leurs avatars, et les lester de leur poids métaphysique, le retour à leur fondement serait utile à une réécriture du commentaire : sans doute de nouveaux savoirs culturels sur le thème de l'Apocalypse, 
sur des mythes comme celui de Babel ou de Sodome, pourraient-ils nourrir l'interprétation du tableau de Kandinsky, dont on connaît l'attachement à la part du Spirituel dans l'art.

Sur Colette, la difficulté du texte est réitérée. Il est difficile à comprendre, à cerner et à décrire. L'élève impute ceci aux mots soutenus, dont il est précisé qu'il y en a énormément : il est fréquent que le seul déficit de la maîtrise lexicale soit identifié par les élèves comme la cause de leurs difficultés de lecture ${ }^{6}$. On peut pourtant penser que la méconnaissance des réalités évoquées pose elle aussi problème (« les terres maraîchères », « ce pays mal pensant était sans danger », ou encore « je revenais à la cloche de la première messe »).

Nous pouvons en conclure que les connaissances culturelles posent certaines difficultés dans les deux commentaires, mais qu'il ne peut être la seule explication des déficits du registre scolaire.

\subsection{Comparaison du registre symbolique}

L'approche de Patrick Rayou et Elisabeth Bautier nous permet de penser que « les apprentissages scolaires ne peuvent (...) se passer du rapport à autrui » et qu'en ce sens tout savoir engage « un registre qui peut être qualifié de symbolique » dans la mesure où il relie à une communauté pour laquelle ce savoir a de la valeur et qu'il « construit un certain type d'identité personnelle » ${ }^{7}$ Ceci nous semble particulièrement tangible concernant les objets qui nous occupent, qui tiennent leur catégorisation comme objets d'art pas seulement de leurs qualités intrinsèques (l'articité d'un objet peut toujours se discuter) mais des décisions d'une communauté autorisée qui les a validés comme tels (en les publiant sous un label littéraire, en les muséographiant, en les inscrivant dans un programme scolaire). Or c'est cette qualité d'objets d'art qui détermine les modalités spécifiques de leur réception, de la construction de leur sens, du moins selon les attendus de l'école : modalités de réception qui n'ont rien d'immédiat ni de naturel, et qui ont peu de choses en commun avec l'appréhension d'un texte ou d'une image dans la vie quotidienne mais aussi avec celle d'un texte qui ne serait pas littéraire ou d'une image qui ne serait pas artistique, dont on ne construit pas les effets de sens des caractéristiques formelles. Entrer dans le jeu de la construction du sens selon la définition scolaire, c'est donc accepter de partager que cette acceptation soit consciente ou non - les valeurs et les catégorisations d'une communauté savante institutionnelle, surplombant les contextes particuliers et les partages horizontaux ${ }^{8}$. Mais au-delà, s'autoriser à jouer le jeu de la construction du sens ainsi défini réclame un engagement identitaire qui est loin d'être anodin. En effet, l'engagement subjectif est nécessaire pour nourrir les opérations cognitives (les prescriptions pédagogiques actuelles, en réaction contre une approche des textes qui fut un temps trop techniciste, incitent les enseignants à favoriser autant que possible cet engagement authentique du sujet lecteur). Simultanément, il faut ressaisir son ressenti, ses émotions, de manière à en abstraire des effets de sens justifiés pour produire un discours qui soit recevable par tout autre, potentiellement universalisable. Comme sur tout autre objet l'école demande aux élèves d'accéder à une inscription de soi dans une autre totalité symbolique que celle du proche, de penser au-delà de soi. Elle est peut-être plus exigeante encore ici, où simultanément elle incite à nourrir cette pensée du plus intime de soi, qu'il sera nécessaire de ressaisir. Du registre symbolique, de l'acceptation de cet engagement de soi, dépend donc finalement qu'il soit possible ou non de jouer le jeu du cognitif, qui suppose d'avoir la capacité de réagir subjectivement, de s'en laisser le droit, mais aussi de s'imposer de retravailler cette réaction.

Dans le commentaire de Laura sur Kandinsky, la mention par deux fois du «peintre » et la voix passive « la façon dont les couleurs sont mises » signalent une perception de l'œuvre comme artefact

\footnotetext{
${ }^{6}$ Goigoux, R. Synthèse de l'étude «Les élèves en grande difficulté de lecture et les enseignements adaptés » (1998), direction des Enseignements scolaires [DESCO], Ministère de l'Éducation nationale, de la Recherche et de la Technologie. Page 151.

7 Bautier, E, Rayou, P, op.cit. Page 144.

${ }^{8}$ Bernstein, B. op cit p. 233.
} 
artistique, contenant en germe du sens. L'acceptation de l'activité de commentateur, composée à la fois d'une impression subjective et de sa ressaisie, est d'emblée en œuvre, puisque l'impression première est donnée puis retravaillée au travers d'une observation de la forme. Ces différents éléments manifestent donc un registre symbolique adéquat aux attendus scolaires.

Cependant, nous avons souligné qu'il manque la justification de plusieurs des interprétations proposées : l'élève reste absorbée dans une réception subjective qui ne se reconfigure pas ou pas encore dans de l'universalisable. Alors que la posture autorisée voudrait qu'elle se mette au service du tableau, l'élève semble presque oublier le tableau pour en faire le prétexte d'un discours sur ellemême et met donc le tableau à son service : ainsi, de l'idée d'un peintre triste ou encore ennervé glisse-t-on immédiatement à un retour sur soi s'appropriant ces affects pour voir le tableau de la façon la plus sombre qui soit : cette idée me fait voir dans le tableau la mort par le rouge du tableau. La tristesse et l'énervement d'abord prêtés arbitrairement au peintre deviennent donc ceux de l'élève... Le passage de comme si le peintre pensait à me fait penser va dans le même sens. Ce retour à soi à travers l'œuvre considérée comme œuvre-miroir, s'il est tout à fait légitime dans un rapport à l'art pour le loisir privé, gêne dans le registre scolaire, du moins il gênerait dans la version finale de l'exercice de commentaire. En revanche, il a sans doute toute sa place dans le processus d'apprentissage, le premier jet qui pourra être réécrit pour que s'activent et s'ajustent progressivement les trois registres d'intellection. Il nous semble très important, pour réussir l'étayage de ce processus, de ne pas réduire ce type de désajustement à l'effet d'une insuffisance du cognitif, mais de l'interroger aussi sur le plan du registre symbolique, puisque la posture face à l'œuvre définie comme souhaitable par la communauté scientifique de référence est partiellement contournée. L'analyse avec l'élève de son propre texte pourrait permettre à un enseignant qui l'aurait préalablement étudié au prisme des registres d'intellection, d'amener l'élève à la conscience des attendus en termes de posture à endosser.

Sur Colette, l'évocation de l'enfance en termes résolument adultes semble perçue comme inaccessible à l'enfant qu'elle est encore. Au-delà de la difficulté de compréhension, un tel traitement de cette période de l'enfance où tout paraît magique (cliché certes, mais cliché auquel le sujet semble adhérer) apparaît à l'élève comme inadéquat : le conditionnel, le texte pourrait parler de cette période, est une expression de l'irréel, auquel s'oppose le présent qui définit la réalité décevante de ce texte qui évoque les choses d'une manière que seul les adultes peuvent évoquer. On peut y lire le refus implicite de jouer le jeu d'un texte qui trahit l'enfance en l'évoquant de façon adulte. Pas d'inscription, donc, dans une communauté de réception du texte, pas de participation à l'entreprise de co-construction du sens d'un tel texte. De cette entreprise cette élève s'exclut consciemment : réservée à la communauté des adultes l'activité d'interprétation est considérée comme étrangère à son monde et à ses compétences. L'appartenance symbolique semble au contraire aller à une communauté d'élèves confrontés à trop de difficulté, de sorte que l'énonciataire implicite du texte devient le professeur (ou le chercheur) à qui il est expliqué que le travail demandé ne peut être fait correctement (par la faute du texte) : l'énonciateur prend en charge implicitement la communauté juvénile des compagnons d'infortune, ce que marque l'impersonnel (il faut le lire et le relire pour comprendre un peu mieux de quoi il s'agit) et les voix passives (Le texte est assez difficile à comprendre puis Le texte est très difficile à cerner et à décrire), qui généralisent la difficulté de compréhension - du moins aux pairs.

Contraste bien sûr intéressant avec le travail sur le tableau : il semble que pour cette élève l'investissement subjectif ou bien ne nourrit pas le registre cognitif - sur le texte - ou bien l'envahit - sur le tableau.

La différenciation de l'activité de construction du sens déployée sur l'un et l'autre des deux objets, activité qui convoque selon nous les trois registres d'intellection profondément enchâssée, est donc bien illustrée par les commentaires de Laura où ces registres ne s'équilibrent pas du tout de la même façon : sur le tableau de Kandinsky, Laura ressaisit son ressenti à partir de références culturelles encore trop peu universelles mais déjà opérantes et de données objectives analysées, le niveau littératié de l'activité cognitive semble en voie de construction ; même si c'est de façon encore trop peu aboutie, elle met en jeu 
l'identité symbolique attendue : elle se sent compétente, prête à adhérer aux réquisits de la communauté de référence et à y jouer sa partition. Pour le texte de Colette, elle n'entre pas ou à peine dans le jeu de la construction interprétative du sens : se considérant comme incompétente elle se replie sur un procédural scolaire qui tourne à vide et ne rencontre pas vraiment son objet.

\section{Conclusion}

Les commentaires écrits par Laura sont plutôt représentatifs de notre corpus global. ${ }^{9}$

D'une part, Laura, qui est dans une classe dont le professeur de français dit n'avoir travaillé qu' une seule fois sur la peinture dans l'année, ne rencontre pas de problème pour formuler un commentaire sur le tableau. Nos résultats confirment que les élèves qui, pour majorité d'entre eux, ont beaucoup moins souvent travaillé dans l'année sur le commentaire de la peinture que sur le commentaire de la littérature, voire ne l'ont jamais travaillé, transposent dans la grande majorité des cas sur l'un des arts les normes de l'exercice qu'ils ont appris sur l'autre ${ }^{10}$ : la transposition sur la peinture de genres scolaires pratiqués sur la littérature (lecture analytique, commentaire) est donc possible (ce qui ne veut pas dire qu'elle garantit dans tous les cas un gain en termes d'apprentissages).

D'autre part, elle atteint un registre plus littératié sur la peinture que sur la littérature. C'est le cas de la majorité des élèves de notre corpus. Nous avons défini, à partir des attendus explicités par les enseignants, classé les commentaires des élèves selon le niveau d'activation plus ou moins littératié de chaque registre (ce classement s'est opéré à partir de critères observables au travers de caractéristiques langagières ${ }^{11}$ que nous avons définies à partir de l'analyse détaillée de certains commentaires). Nous avons, à l'échelle de l'ensemble du corpus, constaté les différences suivantes :

- Pour chacun des critères correspondant au registre cognitif, les commentaires sur la peinture sont sensiblement plus nombreux que les commentaires sur la littérature à se situer à un niveau adéquat.

- Pour le registre culturel, les élèves sont en revanche plus souvent gênés sur la peinture que sur la littérature par des connaissances défectueuses.

- Enfin, c'est sans doute la différence la plus nette, l'identité symbolique apparaît comme plus souvent en phase avec l'activité de construction du sens, telle qu'elle est attendue à l'école, sur la peinture que sur la littérature : selon notre analyse, la représentation que les élèves ont de la peinture leur permet de construire l'objet comme objet d'art et de se construire eux-mêmes comme sujet de leur réception, plus souvent que la représentation qu'ils ont de la littérature ne le leur permet.

Au total, le registre de travail des élèves est le plus souvent plus littératié sur la peinture que sur la littérature. La doxa du détour promue par les enseignants a donc un fondement de vraisemblance.

Mais la déconstruction de l'activité déployée par les élèves par le biais des trois registres nous permet aussi de proposer des explications à ces différences, que nous développons dans la suite de la recherche. Il nous apparaît que c'est bien parce que les deux arts sont différents que les élèves y réussissent différemment. L'étude des différences entre les modalités de production du sens par le pictural et par le littéraire permet, avec l'appui théorique de la sémiologie ${ }^{12}$, d'expliquer que leurs effets

\footnotetext{
${ }^{9}$ Corpus constitué de 106 couples de commentaires écrits dans les mêmes conditions par les élèves de trois classes de troisième et de trois classes de seconde de l'académie de Créteil sur un des trois tableaux de peinture et sur un des trois extraits littéraires.

10 Ils s'acquittent même plus souvent de la tâche sur la peinture que sur la littérature : le texte littéraire n'est pas traité 19 fois et le tableau de peinture 10 fois.

${ }^{11}$ Ces caractéristiques langagières, que nous avons repérées et qui nous permettent de classer les commentaires en différentes catégories pour chacun de nos critères, ne se réduisent pas à celles qui apparaissent dans l'étude des textes de Laura, qui en donnent néanmoins un bon aperçu.

12 Notamment :

Eco, U La production des signes (1976/1992), Librairie Générale Française.
} 


\section{SHS Web of Conferences}

de sens ne sont pas construits par le récepteur de la même façon, n'étant pas produits de la même façon. En outre, les deux arts n'ont pas été construits socialement et scolairement de la même façon, ce qui implique aussi des écarts quant au positionnement du sujet dans leur réception. Les réussites sur l'un des arts ne peuvent profiter automatiquement à l'autre, il n'y a donc pas de raison pour que les élèves sachent d'eux-mêmes transférer des savoir-faire acquis sur l'un des arts à l'autre puisque l'activité à déployer sur l'un et sur l'autre pour arriver au même type de production discursive n'est pas la même. Ceci amène donc à interroger la pertinence d'une lecture de la peinture qui présupposerait possible la transposition à l'identique sur la peinture les démarches du commentaire littéraire et remet en question la doxa du détour. Tout du moins, sa réussite nécessite-t-elle de l'enseignant qu'il explicite les passages possibles, en faisant repérer aux élèves ce qu'ils savent déjà faire dans les différents registres sur l'un des deux arts, et en les aidant à comprendre comment ces savoir-faire peuvent être transposés sur l'autre.

\section{Annexes}

Vassily Kandinsky. Paysage sous la pluie 1913 Huile sur toile 70,2 sur 78,1 Guggenheim Museum, New York.

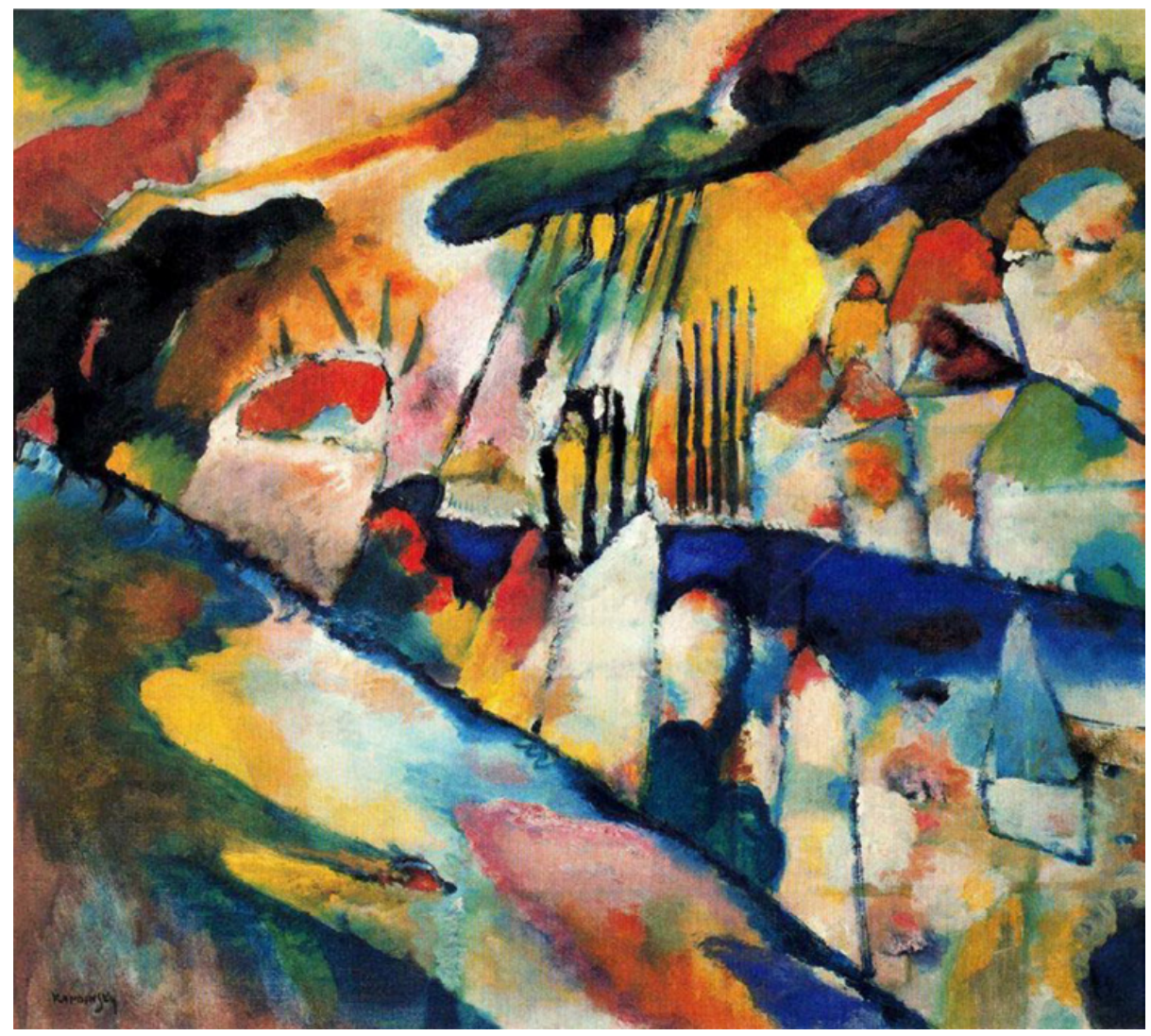

Sido (1929) Colette (1873-1954).

Marin, L Etudes sémiologiques Ecritures, peinture (1971). Klincksieck.

Marin, L Détruire la peinture (1977), Galilée.

Marin, L, De la représentation (1994), Gallimard/Seuil.

Groupe $\mu$, Traité du signe visuel. Pour une rhétorique de l'image (1992) Éditions Le Seuil, Paris. 
Chapeau d'un manuel (seconde). Dans ce court texte, construit autour de la figure rayonnante de sa mère, Sido, Colette retourne à la source de son écriture : l'évocation du milieu familial, l'enfance campagnarde à Saint-Sauveur-en-Puisaye. La grâce de l'enfance retrouvée et le bonheur de la fusion avec la nature s'expriment sans une langue pleine de sensualité.

Car j' aimais tant l'aube, déjà, que ma mère me l'accordait en récompense. J'obtenais qu'elle m'éveillât à trois heures et demie, et je m'en allais, un panier vide à chaque bras, vers des terres maraîchères qui se réfugiaient dans le pli étroit de la rivière, vers les fraises, les cassis et les groseilles barbues. À trois heures et demie, tout dormait dans un bleu originel, humide et confus, et quand je descendais le chemin de sable, le brouillard retenu par son poids baignait d'abord mes jambes, puis mon petit torse bien fait, atteignait mes lèvres, mes oreilles et mes narines plus sensibles que tout le reste de mon corps... J'allais seule, ce pays mal pensant était sans dangers. C'est sur ce chemin, c'est à cette heure que je prenais conscience de mon prix, d'un état de grâce indicible et de ma connivence avec le premier souffle accouru, le premier oiseau, le soleil encore ovale, déformé par son éclosion...

Ma mère me laissait partir, après m'avoir nommée « Beauté, Joyau-tout-en-or »; elle regardait courir et décroître sur la pente son œuvre, - « chef-d'œuvre », disait-elle. J'étais peut-être jolie ; ma mère et mes portraits de ce temps-là ne sont pas toujours d'accord ... Je l'étais à cause de mon âge et du lever du jour, à cause des yeux bleus assombris par la verdure, des cheveux blonds qui ne seraient lissés qu'à mon retour, et de ma supériorité d'enfant éveillée sur les autres enfants endormis. Je revenais à la cloche de la première messe. Mais pas avant d'avoir mangé mon saoul, pas avant d'avoir, dans les bois, décrit un grand circuit de chien qui chasse seul, et goûté l'eau de deux sources perdues, que je révérais. L'une se haussait hors de la terre par une convulsion cristalline, une sorte de sanglot, et traçait elle-même son lit sableux. Elle se décourageait aussitôt née et replongeait sous la terre. L'autre source, presque invisible, froissait l'herbe comme un serpent, s'étalait secrète au centre d'un pré où des narcisses, fleuris en ronde, attestaient seuls sa présence. La première avait goût de feuille de chêne, la seconde de fer et de tige de jacinthe... Rien qu'à parler d'elles je souhaite que leur saveur m'emplisse la bouche au moment de tout finir, et que j'emporte, avec moi, cette gorgée imaginaire... 